VIII.

\title{
Kritische Bemerkungen zu der Breslauer Statistik des Krebses beider Ehegatten und der Frage des Krebses der Ehegatten ïberhaupt.
}

\author{
Von
}

Dr. Weinberg (Stuttgart).

In seiner Veröffentlichung: „Ueber die in den Jahren 1876--1900 in Breslau vorgekommenen Todesfälle an Krebs", behandelt Dr. F. Frief ${ }^{1}$ ) die Frage der Doppelerkrarkungen an Krebs bei Eheleuten vom Standpunkt der Wahrscheinlichkeitsrechnung und gelangt zu dem Ergebnis, dass nach dem Tode Krebskranker von deren überlebenden Ehegatten in 1. Jahre 3 mal, in den ersten 5 Jahren doppelt soviele an Krebs starben, als nach der Wahrscheinlichkeitsrechnung zu erwarten gewesen wäre. Wenn dieses Ergebnis durch eine statistisch einwandfreie Methode gewonnen wäre, so müsste damit dem Krebs eine ebenso grosse Ansteckungsfähigkeit zugeschrieben werden, wie der Tuberkulose (Lungenschwindsucht).

Bei einer Untersuchung der Tuberkulose in Stuttgart, die demnächst zur Veröffentlichung gelangen wird, fand ich nämlich bei den überlebendeı Ehegatten der an Lungenschwindsucht Gestorbenen im 1. Jahre der Witwerschaft 2,7 mal, in den ersten 5 Jahren 2,4 mal soviele schwindsüchtig, als die Wahrscheinlichkeitsrechnung unter Berücksichtigung des Alters des ïberlebenden Ehegatten ergab. Diese Zahlen stimmen mit den von Frief in Breslau für den Krebs bei den Ehegatten gefundenen sehr nahe übereis.

Allein bei näherer Prüfung der Arbeit von Frief ergibt sich, dass diese Zahlen keineswegs statistisch einwandfrei gewonnen sinfl. So liegt seiner Berechnung der tatsächlichen Häufigkeit des Krebses beider Ehe-

1) F. Frief, Die in den Jahren 1876-1900 in Breslau vorgekommenen Todesfälle an Krebs, mit besonderer Berücksichtigung örtlicher Einflüsse auf diese Krankheit. (Aus dem hygienischen Institut zu Breslau.) Klinisches Jahrbuch. Bd. XII. 1904. Verlag von G. Fischer. 
gatten innerhalb des Zeitraums von 5 Jahren eine Anzahl von 73 eingetroffenen Todesfällen zu Grunde (S. 62), während er doch überhaupt von 1876-1900 nur 65 Fälle von Cancer à deux aufführt (S. 52-54) und von diesen fallen nur 21 in den speziell bezüglich der Frage des Cancer à deux untersuchten Zeitraum von 1891-1900'). Die Zahl 73 ist also unrichtig. Ebenso unrichtig wie diese Angabe über die tatsächliche Zahl sind auch die von ihm berechneten wahrscheinlichen Verhältniszahlen, und zwar beruht ihr Fehler darin, dass eine gänzlich falsche Wahrscheinlichkeitsberechnung vorgenommen wurde.

Frief geht nämlich (S. 57-62) von dem Gedanken aus, dass die Wahrscheinlichkeit für die Erkrankung beider Ehegatten gleich ist dem Produkt der beiden Wahrscheinlichkeiten des Erkrankens (bezw. Sterbens) der Männer und Frauen (S. 58). Die so gewonnene Zahl wäre nun, alles übrige als richtig vorausgesetzt, um die Hälfte zu niedrig, da es sich um die Wahrscheinlichkeit eines Erkrankens eines Ehepaars, also zweier Personen handelt; während seine Formel nur für eine Person gilt. Es kann sowohl der Mann zuerst und dann die Frau, als umgekehrt die Frau und dann der Mann an Krebs sterben. Weiterhin sind auch die Formeln falsch, welche Frief für die Wahrscheinlichkeit des Erkrankens der Männer und der Frauen aufgestellt hat. Aus einem Vergleich der (S. 56, 57 und 58) von ihm angegebenen Zahlen der Krebstodesfälle geht hervor, dass der Zähler seiner Formeln je aus der Summe der Verheirateten und Verwitweten besteht, während der Nenner aus der Zahl der Ehepaare besteht. Es starben nämlich z. B. 1891-1895 416 verheiratete Frauen, während er insgesamt 837 Frauen für diese Zeit in seine Formeln einsetzte, und die Zahl der verheirateten + verwitweten Frauen 863 betrug; unter den Verwitweten sind wohl auch die Geschiedenen einbegriffen, deren Zahl wohl gleich der Differenz von 863 und 837 sein dürfte.

Es wird einleuchten, dass hier gänzlich disparate Zahlen in Beziehung gesetzt worden sind und dass diese Formeln keine Sterblichkeitsziffer und keine Wahrscheinlichkeit irgend einer Art vorstellen können. Vielleicht ist die Formel dadurch entstanden, dass Frief sich mit Recht sagte, dass die überlebenden Ehegatten der an Krebs Gestorbenen Witwer und nicht verheiratet sind und dass sich ihre Sterbenswahrscheinlichkeit nicht einfach mit derjenigen der Verheirateten deckt. Allein dieser Tatsache müsste auf ganz andere Weise Rechnung getragen werden.

Die Sterblichkeit an Krebs hängt bekanntlich sehr wesentlich vom Alter ab. Nun ist der Altersaufbau der überlebenden Ehegatten Krebstoter ein ganz spezifischer, sowohl von dem der Verheirateten, wie dem der

1) Warum hat übrigens Frief nicht den ganzen Zeitraum von 1876-1900 untersucht? Das hätte doch seine kleinen absoluten Zahlen wesentlich vergrössert! 
Witwen überhaupt verschiedener. Er muss für jede Untersuchung besouders bestimmt werden und ohne seine Kenntnis ist es überhaupt unmöglich, die wahrscheinliche Sterbeziffer der ïberlebenden Ehegatten Krebstoter zu berechnen.

Die wahrscheinliche Häufigkeit der Fälle von Cancer à-deux hatte Frief annäbernd richtig erhalten durch Addition zweier Produkte, je bestehend aus der Sterbenswahrscheinlichkeit an Krebs für die Verheirateten des einen und der spezifischen Sterblichkeitswahrscheinlichkeit der überlebenden Ehegatten des anderen Geschlechts. Um diese Zahl zu erhalten, hätte für jedes Alter und Geschlecht die Zahl der tatsächlich vorhandenen überlebenden Ehegatten multipliziert werden müssen mit der diesem Alter entsprechenden Krebssterbeziffer und die Summe aller dieser Produkte hätte mit der Zahl sämtlicher überlebender Ehegatten dividiert werden müssen.

$\mathrm{Zu}$ den fundamentalen Fehlern der Untersuchungsmethode Friefs, die vollständig erschöpfend darzustellen zu weit führen würde, kommt nun weiter noch hinzu, dass er den Einfluss der Wanderungen auf die Zahl der Todesfälle nicht berücksichtigt hat. Man muss wissen, wie viele überlebende Ehegatten Krebstoter am Ort der Untersuchung an bestiminten Zeitpunkten nach dem ersten Todesfall an Krebs wohnten; denn man kann nur die Wahrscheinlichkeit des Sterbens am Ort selber berechnen, während man die Wahrscheinlichkeit des Sterbens überhaupt ohne Berücksichtigung. des Orts nur durch mühevolle Erkundigungen und bei genügender Kenntnis der Krebssterblichkeit an allen, ron den hinterbliebenen Ehegatten aufgesuchten Orten ermitteln könute. Dieser Fehler schwächt die Wirkung der übrigen Fehler etwas ab.

Im Folgenden soll nun untersucht werden, wie ungefähr bei richtiger Methode der Wahrscheinlichkeitsberechnung sich das Verhältnis der Zahlen für erwartete und eingetroffene Fälle von Krebs beider Ehegatten gestaltet hätte. $\mathrm{Zu}$ diesem $\mathrm{Zweck}$ muss ich auf das Naterial meiner Untersuchungen über den Krebs beider Ehegatten in Stuttgart zurückgreifen ${ }^{1}$ ). Hier fand ich für den ganzen untersuchten Zeitraum von i873-1902 auf 46 erwartungsmässige 48 eingetroffene Todesfälle an Krebs beider Ehegatten, ein Ergebnis, das mit dem scheinbaren Ergebnis der Friefschen Untersuchung in auffallendem Widerspruch steht. Allerdings hatte ich darauf verzichtet, die erwartungsmässige Sterblichkeit an Krebs in den fünf ersten

1) Weinberg und Gastpar, Die bösartigen Neubildungen in Stuttgart 1873 bis 1902. I. Weinberg, Die Krebstoten; Gastpar, Erkrankung zweier Ehegatten an Neubildung. Zeitschr. f. Krebsforschung. Bd. II. 1904. S. 195-260. Fortsetzung in diesem Heft S. $18-32$. 
Witwerjahren besonders zu berechnen. Indes luätte ich von einer bedeutenden Jebersterblichkeit in diesen ersten fünf Jahren eine wesentliche Erhöhung der tatsächlichen Zahl der Todesfälle in dem gesamten untersuchten Zeitraum deshalb finden müssen, weil die durchschnittliche Dauer der Beobachtung der überlebenden Ehegatten 8 Jahre betrug. Umgekehrt könnte ich aus der nahen Uebereinstimmung zwischen Erwartung und Erfahrong überhaupt schliessen, dass in den fünf ersten Witwerjahren keine erhebliche Uebersterblichkeit an Krebs bestand. Ich habe nun aber diese Berechnung für die Jahre 1893-1902 nachgeholt, um sie mit den Zahlen, die Frief für Breslau gefunden hat, zu vergleichen. Ich gebe zunächst eine Uebersicht über den Altersaufbau der überlebenden Ebegatten der 1893-1902 an Krebs gestorbenen Personen, soweit erstere in Stuttgart wohnen blieben.

Der durchschnittliche Bestand ${ }^{1}$ ) an überlebenden Ehegatten Krebstoter betrug:

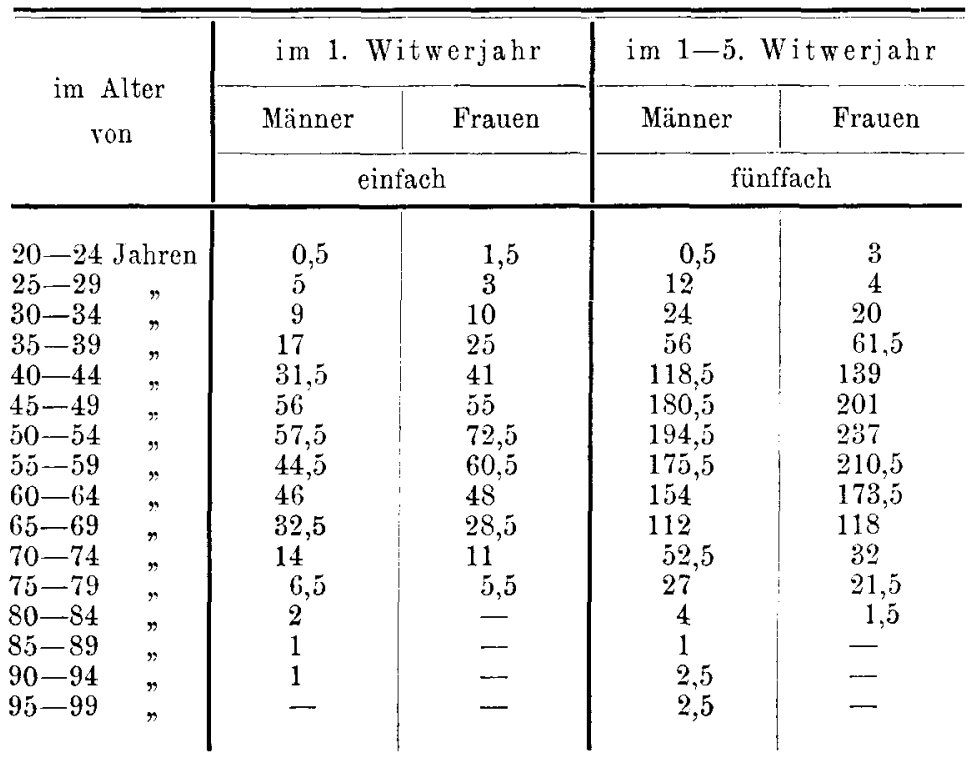

1) Die Berechnung fand derart statt, dass das Alter des Ueberlebenden beim Tode des Ehegatten ermittelt wurde. Standen dann z. B. 27 zu dieser 'Leit im Alter von 35 Jahren und starben und verzogen innerhalb des ersten Witwerjahrs zusammen 7 Personen, so standen am Ende des ersten Witwerjahrs 20 Personen im Alter von 36 Jahren. Der Durchschnitt der Zahlen für Anfang und Ende des ersten Witwerjahrs ergab den Durchschnittsbestand zu dieser Zeit. Diese Borechnungsmethode ist mit der ursprünglich in meiner ersten Arbeit angewandten nicht identisch, sondern noch genauer als erstere. 
Fs starben 1893-1902 an bösartiger Neubildung:

\begin{tabular}{|c|c|c|c|c|c|c|c|c|c|}
\hline \multicolumn{2}{|c|}{$\begin{array}{c}\text { im Alter } \\
\text { von }\end{array}$} & \multicolumn{4}{|c|}{ Männer } & \multicolumn{4}{|c|}{ Frauen } \\
\hline & & & & & bende & & & & bende \\
\hline $20-24$ & Jahren & 5 & auf & $\mathrm{ch}$ & 11975 & 3 & auf & $\operatorname{ch} x$ & 11162 \\
\hline $25-29$ & , & 4 & $\eta$ &. & 8335 & 9 & " & , & 8967 \\
\hline $30-34$ & $m$ & 7 & $"$ & $y$ & 6390 & 22 & $"$ & $n$ & 6854 \\
\hline $35-39$ & , & 20 & $"$ & r & 4979 & 33 & $"$ & $"$ & 5703 \\
\hline $40-44$ & 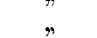 & 20 & $"$ & 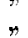 & 4338 & 79 & $"$ & $"$ & 4951 \\
\hline $45-49$ & $"$ & 51 & $"$ & , & 3724 & 109 & $"$ & " & 4257 \\
\hline $50-54$ & $"$ & 97 & " & , & 3243 & 113 & 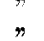 & $"$ & 4133 \\
\hline $55-59$ & 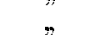 & 92 & 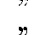 & ... & 2579 & 109 & $"$ & , & 3342 \\
\hline $60-64$ & , & 104 & 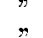 & ". & 1789 & 133 & $"$ & $"$ & 2517 \\
\hline $65-69$ & $"$ & 81 & $"$ & . & 1081 & 109 & $"$ & $"$ & 1677 \\
\hline $70--74$ & " & 58 & 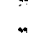 & $r$ & 678 & 80 & 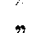 & $r$ & 1113 \\
\hline $75-79$ & , & 26 & , & 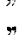 & 337 & 53 & $"$ & $\eta$ & 583 \\
\hline $80-99$ & 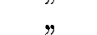 & 12 & " & , & 168 & 28 & $"$ & $n$ & 349 \\
\hline
\end{tabular}

Multipliziert man nun die Zahl der Krebsfälle jeden Alters und Geschlechts mit den entsprechenden Zahlen für den Bestand und dividiert mit der 10 fachen Volkszahl, so erhält man als erwartungsmässige Todesfälle an Neubildung folgende Ziffern:

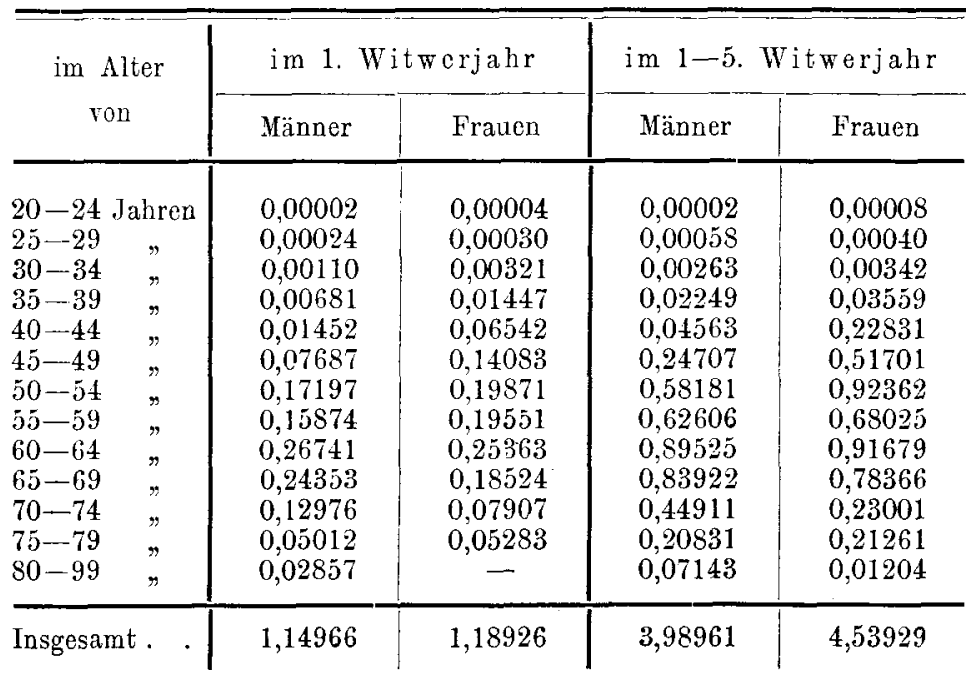

Man erhält also für Stuttgari erwartungsmässige Todesfälle:

im 1. Witwerjahr 2,33 gegen 2 eingetroffene,

" 1 - 5 . " $\quad 8,53 \quad$ " 8 
Nun starben in Stuttgart 1893-1902 445̃ bezw. 407 verheiratete Jänner bezw. Frauen, zusammen 852, in Breslau 1891-1900 860 bezw. 859 verheiratete Männer bezw. Franen, zusammen 1719, oder etwas mehr als doppelt so viel wie in Stuttgart. Dabei hatte Stuttgart 1893-1902 64785 Einwolner im Alter von mehr als 30 Jahren (confer. Frief), hingegen Breslau 152696 Einwohner. Man musste also ceteris paribus diesem Zahlenverhältnis entsprechend die eben gefundenen erwartungsmässigen Zahlen erhöhen, um die erwartungsmässigen Zahlen für Breslau zu erhalten. Diese würden also 5,49 und 20,10 betragen, denen tatsächlich 8 bezw. 21 Fälle von Cancer à deux entsprechen. Demnach wäre die Sterblichkeit der überlebenden Ehegatten Krebstoter im 1. Jahr nicht mehr dreifach, sondern nur 1,5 mal grösser und im 1-5. Jahr nicht doppelt so hoch, sondern nur 1,04 mal grösser, als die Wahrscheinlichkeitsrechnung ergibt. Diesem Resultat steht mein negatives gegenüber, indem auf 2,33 erwartungsmässige nur 2 tatsächliche Fäle von Krebs bei überlebenden Ehegatten im 1. Jahr und entsprechend auf 8,53 erwartungsmässige Fälle im 1-5. Jahr nur 8 tatsächliche Fälle kommen. Vereinigt man beide Ergebnisse, so wäre in den ersten 5 Jahren die tatsächliche Sterblichkeit $\frac{29}{28,63}=1,01 \mathrm{mal}^{1}$ ) grösser, als die Erwartung.

Die von mir angewandte Methode ist der Lebensversicherungstechnik entlehnt und eine andere führt nicht zum richtigen Ziel. Hätte ich nach der Methode Friefs gearbeitet, so hätte ich ebenfalls, wie ich mich überzeugte, eine Sterblichkeit ron ca. $200 \mathrm{pCt}$. der Erwartung erhalten. Dies dürfte an besten den Unterschied der gewählten Methoden beweisen.

Bis jetzt also ist von einem Vergleich der fraglichen Infektiosität des Krebses mit der der Tuberkulose nicht entfernt die Rede.

Sowobl die erwartungsmässigen, wie die absoluten Ziffern sind aber in beiden Untersuchungsreiben so klein, dass der Zufall eine grosse Rolle spielen konnte.

Ich darf aber folgendes hinzufügen: Wenn 48 Fälle an Krebs beider Ehegatten in Stuttgart der Erwartung fast vollständig entsprechen, so werden 65 Fälle in dem über doppelt so grossen Breslau auch unter Berücksichtigung der Tatsache, dass dort die Sterblichkeit an Krebs geringer war und die beobachtete Zeit 5 Jahre weniger umfasste, nicht wesentlich über der erwartungsmässigen Ziffer stehen können. Diese Betrachtung entspricht dem Ergebnis der bisherigen Berechnungen. Beide Statistiken sprechen mit im ganzen 113 Todesfällen gegen den Cancer à deux.

Ich sehe jedoch in der Arbeit von Frief lediglich eine Bestätigung

1) Bei genauerer Berücksichtigung des Altersaufbaus und der Sterblichkeitsunterschiede dürfte vielleicht sogar $1,1-1,2$ herauskommen. 
meiner anderweit bereits ausgesprochenen Ansicht, dass die Frage der Infektiosität des Krebses durch statistische Cntersuchung des Krebses beider Ehegatten nur auf Grund einer viele Jahre im ganzen Deutschen Reich durchgeführten, ebenso mühevollen, wie kostspieligen Statistik zu lösen wäre. Die Vorbedingung des Erfolges einer solchen Statistik, welche unter allen Umständen für Stadt und Land getrennt durchgeführt werden müsste, wäre aber eine exaktere Art der amtlichen Ermittlung der Todesfälle überhaupt. Vorläufig fehlt es in einem grossen Teile des Deutschen Reiches noch an von Aerzten ausgestellten Totenscheinen und an einer genügenden Wahrung des ärztlichen Geheimnisses. Es würde sich also zunächst darum handeln, die Vorbedingungen für die Zulässigkeit einer, das ganze Deutsche Reich umfassenden Statistik zu schaffen. Uebrigens dürfte man einen negativen Ausfall einer solchen Statistik, mit dem entschieden gerechnet werden darf, keineswegs in dem Sinne deuten, als wenn damit die Infektiosität des Krebses völlig widerlegt wäre. Im ganzen ist man in der Lmgebung Krebskranker bedeutend vorsichtiger, als Tuberkulösen gegenüber. Ein negatives Ergebnis würde also lediglich beweisen, dass die Infektion in den meisten Fällen tatsächlich vermieden wird und dass eine staatliche Fürsorge gegen den Krebs unnötig ist ${ }^{1}$ ).

Hingegen wird ein positives Resultat nicht ohne weiteres im Sinne einer direkten Infektion gedeutet werden können, es kann sich, wie Frief in massroller Deutung seiner scheinbaren Ergebnisse herrorhebt, ebenso gut um eine gemeinsame (äussere) Schädlichkeit handeln ${ }^{2}$ ). Nur wenn sich unter Berücksichtigung des Altersaufbaus bei den Ehegatten Krebstoter eine wesentlich grössere Lebersterblichkeit herausstellte, als bei den Bewohnern der entsprechenden Häuser überhaupt, würde man die direkte Infektion als erwiesen betrachten dürfen. Dazu wäre aber nötig, dass fortwährend Buch über die Einwohner jedes Hauses geführt würde mit einer Ausführlichkeit und Genanigkeit, welcher die meisten Einwohnermeldeämter und Wohnungsämter bis jetzt nicht zu entsprechen im Stande sind. Auch eine ärztliche Meldepflicht für Krebs wäre vielleicht notwendig.

Was sollen gegenüber diesen Erwägungen eine Sammelforschung bedeuten, welche die Kranken zum Gegenstand haben, eines Vergleichsobjektes entbehren und an denen dem einzelnen Arzt freisteht, sich zu beteiligen, und an denen er sich besonders gern dann beteiligt, wenn er

1) Siehe auch die in diesem Heft erscheinende Arbeit von Weinberg und Gastpar äber die lokale Verteilung der bösartigen Neubildungen in Krebshäusern, S. $22-32$.

2) Die vielerörterte Feuchtigkeit des Bodens und der Häuser kann auch ohne Parasiten den Krebs befördern, indem sie durch Erkältungskrankheiten zu Anämie führt und die Widerstandsfähigkeit des Körpers untergräbt. 
positive Meldungen zu bringen hat, während negative Fälle als uninteressant bescheiden verschwiegen werden. Solche Sammlungen sind keine Statistik, sie dienen nur zur unbewussten Fälschung der Statistik, so lange das Verständnis für Statistik bei den meisten Aerzten noch wenig entwickelt ist. Die unvollkommenste Statistik der Totenscheine eines ganzen Bezirks ist mehr wert, als diese freiwilligen Sammlungen ${ }^{1}$ ).

Einen Erfolg wird nur eine genaue Methode aus zuverlässigem, in unbefangener Weise gewonnenem, sehr grossem Material ermöglichen, wobei das negative Ergebnis ebenso hocb zu schätzen ist, wie das positive. Bis jetzt ist es aber der Statistik des Cancer à deux nicht gelungen, dem experimentellen Nachweis des immerhin noch überhaupt fraglichen Krebsparasiten vorzugreifen.

1) Siehe auch meine erst nach dieser Arbeit abgefassten Thesen für die ätiologische Statistik des Krebses. Münchener med. Wochenschr. 1905. No. 50. 